\title{
New insights on weighted pseudo almost periodic solutions in a Lasota-Wazewska system
}

\author{
Changjin $\mathrm{Xu}^{1 *}$, Maoxin Liao ${ }^{2}$, Peiluan $\mathrm{Li}^{3}$ and Shuai Yuan ${ }^{4}$
}

"Correspondence: xcj403@126.com ${ }^{1}$ Guizhou Key Laboratory of Economics System Simulation, Guizhou University of Finance and Economics, Guiyang, P.R. China Full list of author information is available at the end of the article

\begin{abstract}
We study the weighted pseudo almost periodic solutions of a Lasota-Wazewska system. With the aid of fixed point theory and differential inequality strategies, we give a set of new sufficient criteria that guarantee the existence and global exponential stability of weighted pseudo almost periodic solutions to a Lasota-Wazewska system. The obtained results of this manuscript are completely innovative and complement the work of Shao (Appl. Math. Lett. 43:90-95, 2015) to some degree. So far, no scholars have investigated this aspect.
\end{abstract}

MSC: 34C25; 34C13; 34C20; 34K25; $92 \mathrm{~B} 20$

Keywords: Weighted pseudo almost periodic solution; Lasota-Wazewska system; Global exponentially stability; Time delay

\section{Introduction}

In 1988, Wazewska-Czyzewska and Lasota [2] proposed the delayed differential model

$$
\dot{w}(t)=-\varrho(t) w(t)+\sum_{k=1}^{p} \theta_{k}(t) e^{-\eta_{k}(t) w\left(t-\rho_{k}(t)\right)}
$$

to describe the survival of red cells in an animal [1]. In this model, $p$ is a positive integer, $w(t)$ stands for the number of red blood cells at time $t, \varrho(t)$ stands for the death rate of the red blood cell, $\theta_{k}(t)$ and $\eta_{k}(t)$ are related to the production of red blood cells per unit time, and $\rho_{k}(t)$ represents the time to produce a red blood cell. For details, see [2].

We know that the death rate or harvesting rate usually change under different seasonal fluctuations. In addition, the actual living environment of species have weighted pseudo almost periodic nature due to the effect of human activities and industrial production, for example, the exhaust emission and reconstruction of rivers. Based on this viewpoint, we think that it is reasonable to suppose that the coefficients in model (1.1) are weighted pseudo almost periodic functions, which can be expressed as an almost periodic component plus the weighted ergodic perturbation. Thus a key problem aries: seek the new suf-

(c) The Author(s) 2020. This article is licensed under a Creative Commons Attribution 4.0 International License, which permits use, sharing, adaptation, distribution and reproduction in any medium or format, as long as you give appropriate credit to the original author(s) and the source, provide a link to the Creative Commons licence, and indicate if changes were made. The images or other third party material in this article are included in the article's Creative Commons licence, unless indicated otherwise in a credit line to the material. If material is not included in the article's Creative Commons licence and your intended use is not permitted by statutory regulation or exceeds the permitted use, you will need to obtain permission directly from the copyright holder. To view a copy of this licence, visit http://creativecommons.org/licenses/by/4.0/. 
ficient conditions to ensure the existence and exponential stability of the weighted pseudo almost periodic solution for model (1.1).

Unfortunately, until now, no scholars have considered the weighted pseudo almost periodic solutions for model (1.1). To make up for this deficiency and inspired by the previous discussion, in this work, we concentrate on the weighted pseudo almost periodic solutions for model (1.1).

Let $\mathcal{B C}(R, R)$ denote the set of all bounded continuous functions from $R$ to $R$, and let $\bar{g}=$ $\sup _{t \in R} g(t)$ and $g=\inf _{t \in R} g(t)$ for a bounded continuous function $g(t)$. The initial condition of system (1.1) is given by

$$
w(s)=\psi(s), \quad s \in[-\bar{\rho}, 0],
$$

where $\psi \in \mathcal{B C}\left([-\bar{\rho}, 0], R^{+}\right), \rho=\max _{k=1,2, \ldots, p} \bar{\rho}_{k}$, and $R^{+}$denotes the nonnegative real numbers.

We plan the paper as follows. In Sect. 2, we present some preliminary knowledge on weighted pseudo almost periodic solution. In Sect. 3, we investigate the existence and global exponential stability of weighted pseudo almost periodic solution to model (1.1). In Sect. 4, numerical simulations are put into effect. The conclusion is given to end this paper in Sect. 5.

\section{Basic knowledge}

Throughout this manuscript, let $\mathcal{U}$ denote the collection of functions (weights) $v: R \rightarrow$ $(0,+\infty)$, which are locally integrable on $R$ and satisfy

$$
\lim _{\gamma \rightarrow+\infty} v([-\gamma, \gamma])=+\infty, \quad \text { where } v([-\gamma, \gamma])=\int_{-\gamma}^{\gamma} v(s) d s \quad(\gamma>0) .
$$

Let $\|w\|=|w|, g^{+}=\sup _{t \in R}|g(t)|, g^{-}=\inf _{t \in R}|g(t)|, \mathcal{U}_{\infty}=\left\{v \mid v \in \mathcal{U}, \inf _{t \in R} v(w)=v_{0}>0\right\}$, $\mathcal{U}_{\infty}^{+}=\left\{v \mid v \in \mathcal{U}, \lim _{|w| \rightarrow+\infty} \sup \frac{v(a w)}{v(w)}<+\infty, \lim _{\gamma \rightarrow+\infty} \sup \frac{v([-a \gamma, a \gamma])}{v([-\gamma, \gamma])}<+\infty\right.$ for $\left.a \in(0,+\infty)\right\}$ Denote the set of bounded continuous function from $R$ to $R$ by $\mathcal{B C}(R, R)$. So $(\mathcal{B C}(R, R)$, $\left.\|\cdot\|_{\infty}\right)$ is a Banach space with norm $\|g\|_{\infty}=\sup _{t \in R}\|g(t)\|$. Denote the set of the almost periodic functions from $R$ to $R$ by $\mathcal{A P}(R, R)$. Define

$$
\operatorname{PAP}_{0}^{v}(R, R)=\left\{\phi \in \mathcal{B C}(R, R)\left|\lim _{\gamma \rightarrow+\infty} \frac{1}{v([-\gamma, \gamma])} \int_{-\gamma}^{\gamma}\right| \phi(t) \mid v(t) d t=0\right\} .
$$

We say that a function $g \in \mathcal{B C}(R, R)$ is weighted pseudo almost periodic if $g=f+\phi$, where $f \in A P(R, R)$ and $\phi \in P A P_{0}^{v}(R, R)$. Here $f$ is called the almost periodic component, and $\phi$ is called the weighted ergodic perturbation. We denote by $P A P^{v}(R, R)$ the space of all weighted almost periodic functions. For more detail, see [3-15].

Lemma 2.1 ([2]) Let $\bar{\varrho}: R \rightarrow(0,+\infty)$ be a bounded continuous function, and let $\alpha^{M}, \alpha^{m}$, $\beta^{M}, \beta^{m}, \sigma, \chi$ be positive constants such that

$$
\begin{aligned}
& \alpha^{m} e^{-\int_{s}^{t} \bar{\varrho}(v) d v} \leq e^{-\int_{s}^{t} \varrho(v) d v} \leq \alpha^{M} e^{-\int_{s}^{t} \bar{\varrho}(v) d v}, \quad \forall t, s \in R \text { and } t-s \geq 0, \\
& -\beta^{M}=\left(-\bar{\varrho}(t) \chi+\alpha^{M} \sum_{k=1}^{r} \theta_{k}(t)\right)^{+}, \quad \chi>\sigma, \\
& -\beta^{m}=\left(-\varrho^{*}(t) \sigma+\alpha^{m} \sum_{k=1}^{r} \theta_{k}(t) e^{-\eta_{k}(t) \chi}\right)^{-} .
\end{aligned}
$$


Then there exists $t_{0}$ such that the solution $w(t)$ of system (1.1) with initial condition (1.2) satisfies $\sigma<w(t)<\chi$ for $t>t_{0}$.

We introduce the following assumptions:

$\left(\mathcal{A}_{1}\right)$ For $k=1,2, \ldots, p, \theta_{k}, \eta_{k}, \rho_{k} \in \mathcal{P} \mathcal{A} \mathcal{P}^{v}(R, R), \varrho \in \mathcal{A} \mathcal{P}(R, R)$,

$$
M[\varrho]=\lim _{\mathcal{T} \rightarrow+\infty} \frac{1}{\mathcal{T}} \int_{t}^{t+\mathcal{T}} \varrho(s) d s>0
$$

$\left(\mathcal{A}_{2}\right) v \in \mathcal{U}_{\infty}^{+}$, and there exist constants $\epsilon>0$ and $\zeta>0$ such that

$$
\sup _{t \in R}\left\{-\bar{\varrho}(t)+\zeta^{-1} \alpha^{M} \sum_{k=1}^{p}\left|\theta_{k}(s)\right|\left|\eta_{k}(s)\right| e^{-\eta_{k}^{-} \sigma}\right\}<-\epsilon .
$$

\section{Main results}

Lemma 3.1 Define the operator

$$
\Gamma(\psi)(t)=\int_{-\infty}^{t} e^{-\int_{s}^{t} \varrho(v) d v}\left[\zeta^{-1} \sum_{k=1}^{p} \theta_{k}(t) e^{-\zeta \eta_{k}(t) \psi\left(t-\rho_{k}(t)\right)}\right] d s, \quad \psi \in \mathcal{P} \mathcal{A P}^{v}(R, R)
$$

Then $\Gamma$ maps $\mathcal{P} \mathcal{A} \mathcal{P}^{v}(R, R)$ into itself.

Proof In view of $\left(\mathcal{A}_{1}\right)$ and Lemmas 2.1 and 2.3 of [16], we have that

$$
\sum_{k=1}^{p} \theta_{k}(t) e^{-\zeta \eta_{k}(t) \psi\left(t-\rho_{k}(t)\right)} \in \mathcal{P} \mathcal{A} \mathcal{P}^{v}(R, R)
$$

According to $\left(\mathcal{A}_{1}\right)-\left(\mathcal{A}_{2}\right)$ and applying the proof of Lemma 2.1 of [17], we can see that $\Gamma(\psi) \in \mathcal{B C}(R, R)$. Then there exist $\mathcal{E}(t) \in \mathcal{A P}(R, R)$ and $\mathcal{F}(t) \in \mathcal{P} \mathcal{A} \mathcal{P}_{0}^{v}(R, R)$ such that

$$
\sum_{k=1}^{p} \theta_{k}(t) e^{-\zeta \eta_{k}(t) \psi\left(t-\rho_{k}(t)\right)}=\mathcal{E}(t)+\mathcal{F}(t)
$$

Since

$$
M[\varrho]=\lim _{\mathcal{T} \rightarrow+\infty} \frac{1}{\mathcal{T}} \int_{t}^{t+\mathcal{T}} \varrho(s) d s>0,
$$

applying the theory of exponential dichotomy of [18], we get that

$$
\int_{-\infty}^{t} e^{-\int_{s}^{t} \varrho(v) d v} \mathcal{E}(s) d s \in \mathcal{A P}(R, R)
$$

is a solution of the following equation

$$
\dot{u}(t)=-\varrho(t) u(t)+\mathcal{E}(t) .
$$

By the proof of Lemma 2.3 of [19] we have that

$$
\int_{-\infty}^{t} e^{-\int_{s}^{t} \varrho(v) d v} \mathcal{F}(s) d s \in \mathcal{P} \mathcal{A} \mathcal{P}_{0}^{v}(R, R) .
$$


In view of (3.1) and (3.3), we conclude that

$$
\Gamma(\psi)(t)=\int_{-\infty}^{t} e^{-\int_{s}^{t} \varrho(v) d v}\left[\zeta^{-1} \sum_{k=1}^{p} \theta_{k}(t) e^{-\zeta \eta_{k}(t) \psi\left(t-\rho_{k}(t)\right)}\right] d s \in \mathcal{P} \mathcal{A} \mathcal{P}^{v}(R, R)
$$

and $\Gamma$ maps $\mathcal{P} \mathcal{A P}^{v}(R, R)$ into itself. This completes the proof.

Theorem 3.1 In addition to the condition of Lemma 2.1, suppose that $\left(\mathcal{A}_{1}\right)-\left(\mathcal{A}_{2}\right)$ are fulfilled. Then model (1.1) has a unique weighted pseudo almost periodic solution, which is globally exponentially stable.

Proof Let $u(t)=\zeta^{-1} w(t)$. Then system (1.1) becomes

$$
\dot{u}(t)=-\varrho(t) u(t)+\zeta^{-1} \sum_{k=1}^{p} \theta_{k}(t) e^{-\zeta \eta_{k}(t) u\left(t-\rho_{k}(t)\right)}
$$

For $\psi, \phi \in \mathcal{P} \mathcal{A} \mathcal{P}^{v}(R, R)$, in view of $\left(\mathcal{A}_{1}\right)$ and $\left(\mathcal{A}_{2}\right)$, we have

$$
\begin{aligned}
\mid & (\Gamma \psi)(t)-(\Gamma \phi)(t) \mid \\
& =\mid \int_{-\infty}^{t} e^{-\int_{s}^{t} \varrho(v) d v}\left[\zeta^{-1} \sum_{k=1}^{p} \theta_{k}(s)\left[e^{-\zeta \eta_{k}(s) \psi\left(s-\rho_{k}(s)\right)}-e^{-\zeta \eta_{k}(s) \phi\left(s-\rho_{k}(s)\right)}\right] d s \mid\right. \\
& \leq \int_{-\infty}^{t} e^{-\int_{s}^{t} \bar{\varrho}(v) d v} \zeta^{-1} \alpha^{M} \sum_{k=1}^{p} \theta_{k}(s) \eta_{k}(s) e^{-\eta_{k}^{-} \sigma}\left|\psi\left(s-\rho_{k}(s)\right)-\phi\left(s-\rho_{k}(s)\right)\right| d s \\
& \leq \sup _{t \in R} \int_{-\infty}^{t} e^{-\int_{s}^{t} \bar{\varrho}(v) d v} \zeta^{-1} \alpha^{M} \sum_{k=1}^{p}\left|\theta_{k}(s)\right|\left|\eta_{k}(s)\right| e^{-\eta_{k}^{-} \sigma} d s\|\psi(t)-\phi(t)\|_{\infty} \\
& \leq \sup _{t \in R} \int_{-\infty}^{t} e^{-\int_{s}^{t} \bar{\varrho}(v) d v}[\bar{\varrho}(s)-\epsilon] d s\|\psi(t)-\phi(t)\|_{\infty} \\
& \leq \sup _{t \in R} \int_{-\infty}^{t} e^{-\int_{s}^{t} \bar{\varrho}(v) d v} d\left(-\int_{s}^{t} \bar{\varrho}(v) d v\right)-\frac{\epsilon}{2} \int_{-\infty}^{t} e^{-\int_{s}^{t} \bar{\varrho}(v) d v} d s\|\psi(t)-\phi(t)\|_{\infty} \\
& \leq\left(1-\frac{\epsilon}{2 \bar{\varrho}^{+}}\right)\|\psi(t)-\phi(t)\|_{\infty}
\end{aligned}
$$

for $l=1,2, \ldots, r$. By $\left(\mathcal{A}_{2}\right)$ we easily see that $\left(1-\frac{\epsilon}{2 \bar{\varrho}^{+}}\right) \in(0,1)$, and thus $\Gamma$ is a contraction mapping. Thus $\Gamma$ has a unique fixed point $u^{*} \in \mathcal{P} \mathcal{A} \mathcal{P}^{v}(R, R)$ and satisfies $\Gamma u^{*}=u^{*}$. Based on this analysis, we conclude that model (1.1) has a unique weighted pseudo almost periodic solution $w^{*}=\zeta u^{*} \in \mathcal{P} \mathcal{A} \mathcal{P}^{v}(R, R)$.

Next, we will prove the exponential stability of the solution $w^{*}$. Let $w(t)$ be an arbitrary solution of (1.1) with initial condition $\psi(t)$ satisfying (1.2). Let $v(t)=\zeta^{-1}\left(w(t)-w^{*}(t)\right)$. Then we have

$$
\dot{v}(t)=-\varrho(t) v(t)+\zeta^{-1} \sum_{k=1}^{p} \theta_{k}(t)\left[e^{-\eta_{k}(t) w\left(t-\rho_{k}(t)\right)}-e^{-\eta_{k}(t) w^{*}\left(t-\rho_{k}(t)\right)}\right] .
$$


Let

$$
\Phi(\varphi)=\sup _{t \in R}\left\{\varphi-\bar{\varrho}(t)+\zeta^{-1} \alpha^{M} \sum_{k=1}^{p}\left|\theta_{k}(s)\right|\left|\eta_{k}(s)\right| e^{-\eta \bar{k}^{-} \sigma} e^{\varphi \rho_{k}(t)}\right\}, \quad \varphi \in\left[0, \bar{\varrho}^{-}\right] .
$$

Then

$$
\Phi(0)=\sup _{t \in R}\left\{-\bar{\varrho}(t)+\zeta^{-1} \alpha^{M} \sum_{k=1}^{p}\left|\theta_{k}(s)\right|\left|\eta_{k}(s)\right| e^{-\eta_{k}^{-} \sigma}\right\} \leq-\epsilon<0 .
$$

Since $\Phi(\varphi)$ is continuous, we can choose a constant $\mu \in\left(0, \bar{\varrho}^{-}\right)$such that

$$
\Phi(\mu)=\sup _{t \in R}\left\{\mu-\bar{\varrho}(t)+\zeta^{-1} \alpha^{M} \sum_{k=1}^{p}\left|\theta_{k}(s)\right|\left|\eta_{k}(s)\right| e^{-\eta_{\bar{k}}^{-} \sigma}\right\}, \quad \varphi \in\left[0, \bar{\varrho}^{-}\right] .
$$

Set

$$
\left\|\psi-w^{*}\right\|_{\zeta}=\sup _{t \in(-\bar{\rho}, 0]} \zeta^{-1}|\psi(t)-w(t)|
$$

and choose $\mathcal{N}>\alpha^{M}+1$. Then we obtain that

$$
\|v(t)\| \leq\left(\left\|\psi-w^{*}\right\|_{\zeta}+\epsilon\right) e^{-\mu t}<\left(\mathcal{N}\left(\left\|\psi-w^{*}\right\|_{\zeta}+\epsilon\right) e^{-\mu t}, \quad t \in(-\bar{\rho}, 0] .\right.
$$

Next, we will prove that

$$
\|v(t)\| \leq\left(\left\|\psi-w^{*}\right\|_{\zeta}+\epsilon\right) e^{-\mu t}<\left(\mathcal{N}\left(\left\|\psi-w^{*}\right\|_{\zeta}+\epsilon\right) e^{-\mu t}, \quad t>0 .\right.
$$

If (3.12) were not true, then there would exist $\xi>0$ such that

$$
\left\{\begin{array}{l}
|v(\xi)|=\mathcal{N}\left(\left\|\psi-w^{*}\right\|_{\zeta}+\epsilon\right) e^{-\mu \xi} \\
|v(t)|=\mathcal{N}\left(\left\|\psi-w^{*}\right\|_{\zeta}+\epsilon\right) e^{-\mu t}, \quad t \in(-\bar{\rho}, \xi)
\end{array}\right.
$$

In view of

$$
\begin{aligned}
\dot{v}(s) & +\varrho(s) v(s) \\
& =\zeta^{-1} \sum_{k=1}^{p} \theta_{k}(s)\left[e^{-\eta_{k}(s) \zeta w\left(s-\rho_{k}(s)\right)}-e^{-\eta_{k}(s) \zeta w^{*}\left(s-\rho_{k}(s)\right)}\right], \quad s \in[0, t], t \in[0, \xi],
\end{aligned}
$$

we have

$$
\begin{aligned}
v(s)= & v(0) e^{-\int_{0}^{t} \varrho(v) d v}+\int_{0}^{t} e^{-\int_{s}^{t} \varrho(v) d v} \\
& \times\left\{\zeta^{-1} \sum_{k=1}^{p} \theta_{k}(s)\left[e^{-\eta_{k}(s) \zeta w\left(s-\rho_{k}(s)\right)}-e^{-\eta_{k}(s) \zeta w^{*}\left(s-\rho_{k}(s)\right)}\right]\right\} d s, \quad t \in[0, \xi] .
\end{aligned}
$$


Hence

$$
\begin{aligned}
& |v(s)|=\mid v(0) e^{-\int_{0}^{t} \varrho(v) d v}+\int_{0}^{t} e^{-\int_{s}^{t} \varrho(v) d v} \\
& \times\left\{\zeta^{-1} \sum_{k=1}^{p} \theta_{k}(s)\left[e^{-\eta_{k}(s) \zeta w\left(s-\rho_{k}(s)\right)}-e^{-\eta_{k}(s) \zeta w^{*}\left(s-\rho_{k}(s)\right)}\right]\right\} d s \mid \\
& \leq \mathcal{N}\left(\left\|\psi-w^{*}\right\|_{\zeta}+\epsilon\right) \alpha^{M} e^{-\int_{0}^{\xi} \bar{\varrho}(v) d v}+\int_{0}^{\xi} e^{-\int_{s}^{\xi} \bar{\varrho}(v) d v} \alpha^{M} \\
& \times\left[\zeta^{-1} \sum_{k=1}^{p}\left|\theta_{k}(s)\right| e^{-\eta \bar{k}^{-} \sigma} \mid v\left(s-\rho_{k}(s) \mid\right] d s\right. \\
& \leq \mathcal{N}\left(\left\|\psi-w^{*}\right\|_{\zeta}+\epsilon\right) \alpha^{M} e^{-\int_{0}^{\xi} \bar{\varrho}(v) d v}+\int_{0}^{\xi} e^{-\int_{s}^{\xi} \bar{\varrho}(v) d v} \alpha^{M} \\
& \times\left[\zeta^{-1} \sum_{k=1}^{p}\left|\theta_{k}(s)\right| e^{-\eta_{k}^{-} \sigma} \mathcal{N}\left(\left\|\psi-w^{*}\right\|_{\zeta}+\epsilon\right) e^{-\mu\left(s-\rho_{k}(s)\right)}\right] d s \\
& \leq \mathcal{N}\left(\left\|\psi-w^{*}\right\|_{\zeta}+\epsilon\right) e^{-\mu \xi} \alpha^{M} e^{-\int_{0}^{\xi}(\bar{\varrho}(v)-\mu) d v}+\int_{0}^{\xi} e^{-\int_{S}^{\xi}(\bar{\varrho}(v)-\mu) d v} \alpha^{M} \\
& \times\left[\zeta^{-1} \sum_{k=1}^{p}\left|\theta_{k}(s)\right| e^{-\eta \bar{k}^{-} \sigma} \mathcal{N}\left(\left\|\psi-w^{*}\right\|_{\zeta}+\epsilon\right) e^{\mu \rho_{k}(s)}\right] d s \mathcal{N}\left(\left\|\psi-w^{*}\right\|_{\zeta}+\epsilon\right) e^{-\mu \xi} \\
& \leq \mathcal{N}\left(\left\|\psi-w^{*}\right\|_{\zeta}+\epsilon\right) e^{-\mu \xi} \alpha^{M} e^{-\int_{0}^{\xi}(\bar{\varrho}(v)-\mu) d v} \\
& +\int_{0}^{\xi} e^{-\int_{s}^{\xi}(\varrho(v)-\mu) d v}(\bar{\varrho}(v)-\mu) d s \mathcal{N}\left(\left\|\psi-w^{*}\right\|_{\zeta}+\epsilon\right) e^{-\mu \xi} \\
& \leq \mathcal{N}\left(\left\|\psi-w^{*}\right\|_{\zeta}+\epsilon\right) e^{-\mu \xi}\left[1-\left(1-\frac{\alpha^{M}}{\mathcal{N}}\right) e^{-\int_{s}^{\xi}(\bar{Q}(v)-\mu) d v}\right] \\
& \leq \mathcal{N}\left(\left\|\psi-w^{*}\right\|_{\zeta}+\epsilon\right) e^{-\mu \xi} \text {. }
\end{aligned}
$$

By (3.13) we get that (3.12) holds. Let $\epsilon \rightarrow 0^{+}$. Then

$$
\|v(t)\|<\mathcal{N}\left(\left\|\psi-w^{*}\right\|_{\zeta}+\epsilon\right) e^{-\mu t}, \quad t>0
$$

Therefore we conclude that the pseudo almost periodic solution of model (1.1) is globally exponentially stable. The proof is finished.

Remark 3.1 Shao [1] studied the pseudo almost periodic solution of model (1.1). In this paper, we considered the weighted pseudo almost periodic solution. All the assumptions in this paper are different from those in [1]. All the derived results in [1] cannot be applicable to system (1.1) to establish the existence and globally exponential stability of weighted pseudo almost periodic solution for model (1.1). Based on this viewpoint, our results on the existence and globally exponential stability of weighted pseudo almost periodic solution for model (1.1) are essentially new and complement earlier works to a certain extent. 


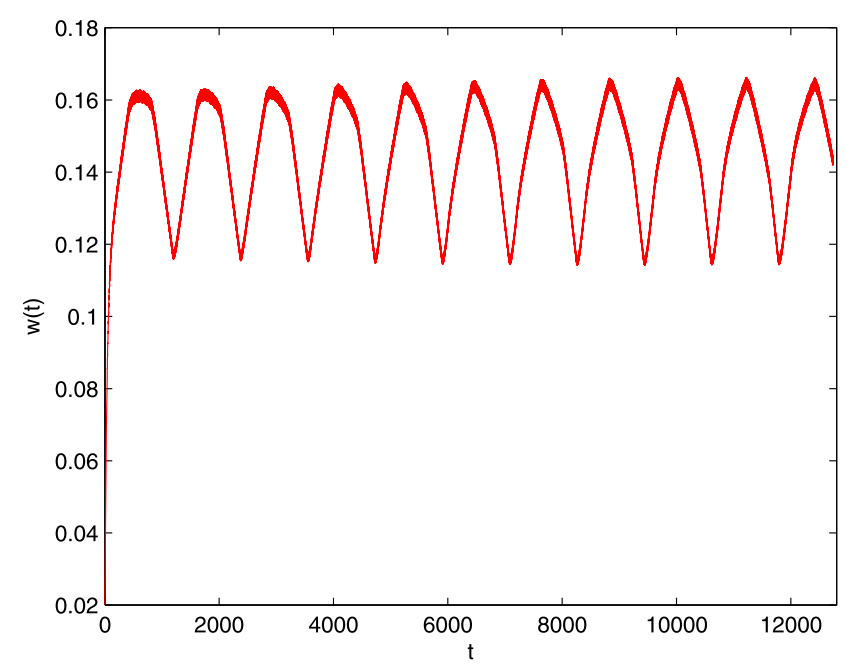

Figure 1 Numerical results of model (4.1): $t-w(t)$

\section{Computer simulations}

Consider the system

$$
\dot{w}(t)=-\varrho(t) w(t)+\sum_{k=1}^{2} \theta_{k}(t) e^{-\eta_{k}(t) w\left(t-\rho_{k}(t)\right)}
$$

where $\varrho(t)=17+10 \cos 1000 t, \theta_{1}(t)=2(1+0.5|\sin 1.7 t|), \theta_{2}(t)=2(0.8+0.2|\sin 1.7 t|)$, $\eta_{1}(t)=-1+0.2|\sin 5 t|, \eta_{2}(t)=-0.9+0.5|\sin 30 t|, \rho_{1}(t)=e^{0.2|\sin t|}, \rho_{2}(t)=e^{0.3|\sin t|}$. Let $\bar{\varrho}(t)=17, M[\varrho]=17, \alpha^{M}=\frac{1}{200}, \alpha^{m}=-\frac{1}{200}, \chi=0.8, \sigma=0.2, \zeta=2, \epsilon=3$. Then

$$
\alpha^{m} e^{-\int_{s}^{t} \bar{\varrho}(v) d v} \leq e^{-\int_{s}^{t} \varrho(v) d v} \leq \alpha^{M} e^{-\int_{s}^{t} \bar{\varrho}(v) d v} \quad \text { for } t, s \in R \text { such that } t-s \geq 0
$$

and

$$
\sup _{t \in R}\left\{-\bar{\varrho}(t)+\zeta^{-1} \alpha^{M} \sum_{k=1}^{p}\left|\theta_{k}(s)\right|\left|\eta_{k}(s)\right| e^{-\eta \bar{k}^{-} \sigma}\right\} \approx-3.087<-3=-\epsilon .
$$

Thus all the hypotheses of Theorem 3.1 are satisfied, and so system (4.1) has a unique weighted pseudo almost periodic solution, which is globally exponentially stable. Figure 1 reveals this fact.

\section{Conclusions}

In this paper, we have discussed the existence and globally exponential stability of weighted pseudo almost periodic solutions for a Lasota-Wazewska system. Using the fixed point theory and differential inequalities, we establish new sufficient criteria ensuring the existence and globally exponential stability of weighted pseudo almost periodic solutions for the Lasota-Wazewska model. The derived results complement some earlier publications to some extent. Up to now, to the best of our knowledge, it is the first time to deal with this aspect. In the near future, we will investigate the pseudo almost automorphic solutions for the Lasota-Wazewska model. 


\section{Acknowledgements}

The authors would like to thank the referees and the editor for helpful suggestions incorporated into this paper.

\section{Funding}

The work is supported by National Natural Science Foundation of China (No. 61673008), Project of High-level Innovative Talents of Guizhou Province ([2016]5651), Major Research Project of The Innovation Group of The Education Department of Guizhou Province ([2017]039), Innovative Exploration Project of Guizhou University of Finance and Economics ([2017]5736-015), Project of Key Laboratory of Guizhou Province with Financial and Physical Features ([2017]004), Hunan Provincial Key Laboratory of Mathematical Modeling and Analysis in Engineering, Changsha University of Science \& Technology (2018MMAEZD21), University Science and Technology Top Talents Project of Guizhou Province (KY[2018]047), Guizhou University of Finance and Economics(2018XZD01), and Foundation of Science and Technology of Guizhou Province ([2019]1051).

\section{Availability of data and materials}

Data sharing not applicable to this paper as no datasets were generated or analyzed during the current study.

\section{Competing interests}

The authors declare that they have no competing interests.

\section{Authors' contributions}

All authors have read and approved the final manuscript

\section{Author details}

'Guizhou Key Laboratory of Economics System Simulation, Guizhou University of Finance and Economics, Guiyang, P.R. China. ${ }^{2}$ School of Mathematics and Physics, University of South China, Hengyang, P.R. China. ${ }^{3}$ School of Mathematics and Statistics, Henan University of Science and Technology, Luoyang, P.R. China. ${ }^{4}$ School of Mathematics and Statistics, Central South University, Changsha, P.R. China.

\section{Publisher's Note}

Springer Nature remains neutral with regard to jurisdictional claims in published maps and institutional affiliations.

Received: 10 March 2020 Accepted: 17 April 2020 Published online: 18 May 2020

\section{References}

1. Shao, J.Y.: Pseudo almost periodic solutions for a Lasota-Wazewska model with an oscillating death rate. Appl. Math. Lett. 43, 90-95 (2015)

2. Wazewska-Czyzewska, M., Lasota, A.: Mathematical problems of the dynamics of red blood cells system. Ann. Pol. Math. 17, 23-40 (1988)

3. Diagana, T.: Weighted pseudo almost periodic solutions to some differential equations. Nonlinear Anal. 68, 2250-2260 (2008)

4. Zhang, T.W., Gan, X.R.: Almost periodic solutions for a discrete fishing model with feedback control and time delays. Commun. Nonlinear Sci. Numer. Simul. 19(1), 150-163 (2014)

5. Zhang, T.W., Li, Y.K., Ye, Y.: On the existence and stability of a unique almost periodic solution of Schoener's competition model with pure-delays and impulsive effects. Commun. Nonlinear Sci. Numer. Simul. 17(3), 1408-1422 (2012)

6. Chen, S.T., Tang, X.H.: On the planar Schrödinger-Poisson system with the axially symmetric potential. J. Differ. Equ. 268, 945-976 (2020)

7. Chen, S.T., Fiscella, A., Pucci, P., Tang, X.H.: Semiclassical ground state solutions for critical Schrödinger-Poisson systems with lower perturbations. J. Differ. Equ. 268(6), 2672-2716 (2020)

8. Chen, S.T., Tang, X.H.: Berestycki-Lions conditions on ground state solutions for a nonlinear Schrödinger equation with variable potentials. Adv. Nonlinear Anal. 9(1), 496-515 (2020)

9. Duan, L., Huang, L.H., Chen, Y.M.: Global exponential stability of periodic solutions to a delay Lasota-Wazewska model with discontinuous harvesting. Proc. Am. Math. Soc. 144, 561-573 (2016)

10. Duan, L., Fang, X.W., Huang, C.X.: Global exponential convergence in a delayed almost periodic Nicholson's blowflies model with discontinuous harvesting. Math. Methods Appl. Sci. 41(5), 1954-1965 (2018)

11. Huang, C.X., Zhang, H., Huang, L.H.: Almost periodicity analysis for a delayed Nicholson's blowflies model with nonlinear density-dependent mortality term. Commun. Pure Appl. Anal. 18(6), 3337-3349 (2019)

12. Huang, C.X., Zhang, H.: Periodicity of non-autonomous inertial neural networks involving proportional delays and non-reduced order method. Int. J. Biomath. 12(2), Article ID 1950016 (2019)

13. Duan, L., Xu, Z.L.: A note on the dynamics analysis of a diffusive cholera epidemic model with nonlinear incidence rate. Appl. Math. Lett. 106, Article ID 106356 (2020)

14. Tang, X.H., Chen, S.T., Lin, X.Y., Yu, J.S.: Ground state solutions of Nehari-Pankov type for Schrödinger equations with local super-quadratic conditions. J. Differ. Equ. 268(8), 4663-4690 (2020)

15. Tang, T.X.H., Chen, S.T.: Singularly perturbed Choquard equations with nonlinearity satisfying Berestycki-Lions assumptions. Adv. Nonlinear Anal. 9(1), 413-437 (2020)

16. Zhou, Q.Y., Shao, J.Y.: Weighted pseudo-anti-periodic SICNNs with mixed delays. Neural Comput. Appl. 29(10), 865-872 (2018)

17. Long, Z.: New results on anti-periodic solutions for SICNNs with oscillating coefficients in leakage terms. Neurocomputing 171(1), 503-509 (2016)

18. Fink, A.M.: Almost Periodic Differential Equations. Lecture Notes in Mathematics, vol. 377. Springer, Berlin (1974)

19. Yang, G.Y., Wan, W.P.: Weighted pseudo almost periodic solutions for cellular neural networks with multi-proportional delays. Neural Process. Lett. 49(3), 1125-1138(2019) 